\title{
ROLE OF SLIP VELOCITY IN A MAGNETO-MICROPOLAR FLUID FLOW FROM A RADIATIVE SURFACE WITH VARIABLE PERMEABILITY: A NUMERICAL STUDY
}

\author{
B.K. SHARMA* \\ Department of Mathematics \\ Birla Institute of Technology and Science \\ Pilani, Rajasthan, INDIA \\ E-mail: bhupen_1402@yahoo.co.in \\ V. TAILOR and M. GOYAL \\ Department of Mathematics \\ University of Rajasthan, Jaipur, Rajasthan, INDIA \\ E-mails: viikas100@gmail.com; mamta1245@gmail.com
}

\begin{abstract}
An analysis is presented to describe the hydromagnetic mixed convection flow of an electrically conducting micropolar fluid past a vertical plate through a porous medium with radiation and slip flow regime. A uniform magnetic field has been considered in the study which absorbs the micropolar fluid with a varying suction velocity and acts perpendicular to the porous surface of the above plate.The governing non-linear partial differential equations have been transformed into linear partial differential equations, which are solved numerically by applying the explicit finite difference method. The numerical results are presented graphically in the form of velocity, micro-rotation, concentration and temperature profiles, the skin-friction coefficient, the couple stress coefficient, the rate of heat and mass transfers at the wall for different material parameters.
\end{abstract}

Key words: slip velocity, chemical reaction, micropolar fluid, MHD, heat and mass transfer, FDM.

\section{Introduction}

Micropolar fluids belong to a class of fluids that exhibit certain microscopic effects arising from the local structure and micro-motions of the fluid elements. Micro-polar fluids contain dilute suspensions of rigid macromolecules with individual motion that support stress and body moments and are influenced by spin inertia. Eringen [1] developed a simple theory (theory of micropolar fluids) which includes the effect of local rotary inertia, the couple stress and the inertial spin. This theory is expected to be useful in analyzing the behavior of non-Newtonian fluids. Eringen [2] extended the theory of thermo-micropolar fluids and derived the constitutive law for fluids with microstructure. This general theory of micropolar fluids deviates from that of the Newtonian fluid by adding two new variables to the velocity. This theory may be applied to explain the phenomenon of the flow of colloidal fluids, liquid crystals, polymeric suspensions, animal blood, etc. Gorla [3] discussed the heat transfer in a micropolar fluid flow over a semi-infinite plate. Rees and Pop [4] studied a free convection boundary layer flow of a micropolar fluid from a vertical flat plate. The flow and heat transfer of a micropolar fluid past a porous plate was studied by Takhar and Soundalgekar [5-6]. Gorla et al. [7-8] investigated further the concept of natural convection from a heated vertical plate in a micropolar fluid.

\footnotetext{
* To whom correspondence should be addressed
} 
Flows of fluids through porous media have attracted the attention of a number of scholars due to their applications in many branches of science and technology, for example in the fields of agriculture engineering to study the underground water resources, in chemical engineering for filtration and purification processes in petroleum technology to study the movement of natural gas, oil, and water through the oil reservoirs. Fluctuating heat and mass transfer on three-dimensional flow through a porous medium with variable permeability has been discussed by Sharma et al. [9]. The problem of flow and heat transfer for a micropolar fluid past a porous plate embedded in a porous medium has been of great use in engineering studies such as oil exploration, thermal insulation, etc. Micropolar fluid flows through a porous medium have been discussed by Raptis and Takhar [10].

There has been a renewed interest in MHD flow with heat and mass transfer due to the important effect of the magnetic field on the performance of many systems using electrically conducting fluids. An unsteady hydromagnetic free convection flow of Newtonian and polar fluids was investigated by Helmy [11]. Hydromagnetic unsteady mixed convection and mass transfer flow past a vertical porous plate immersed in a porous medium has been investigated by Sharma and Chaudhary [12]. Chaudhary and Sharma [13] analysed combined heat and mass transfer by a laminar mixed convection flow from a vertical surface with induced magnetic field. El-Hakien et al. [14] studied the effects of viscous and Joule heating on an MHD-free convection flow with variable plate temperature in a micropolar fluid. An MHD free-convection and mass transfer flow in a micropolar fluid over a stationary vertical plate with constant suction was studied by El-Amin [15]. Kim [16] investigated unsteady free convection flow of a micropolar fluid past a vertical plate embedded in a porous medium and extended to study the effects of heat and mass transfer in an MHD micropolar fluid flow past a vertical moving plate (Kim [17]). Analytical studies on an MHD flow of a micropolar fluid over a vertical porous plate were presented by Kim and Lee [18]. Recently, Sharma et al. [19] studied heat and mass transfer in a magneto-biofluid flow through a non-Darcian porous medium.

Combined heat and mass transfer problems with chemical reaction are of importance in many processes such as drying, evaporation at the surface of water body, energy transfer in wet cooling towers. Das et al. [20] considered the effects of first order chemical reaction on the flow past an impulsively started infinite vertical plate with constant heat flux and mass transfer. Sharma et al. [21-22 ] discussed the effects of chemical reaction on a magneto-micropolar fluid flow from a radiative surface with variable permeability. First order homogeneous chemical reaction on flow past an infinite vertical plate was studied by Muthucumarswamy and Ganesan [23]. Recently, the effect of radiation on an MHD flow and heat transfer problem has become more important industrially. Many processes in engineering areas occur at high temperatures, and a knowledge on radiation heat transfer becomes very important for the design of reliable equipment, gas turbines, nuclear plants and various propulsion devices or aircrafts, missiles, satellites and space vehicles. Sharma et al. [24-25] analysed the radiation effect with simultaneous thermal and mass diffusion in an MHD mixed convection flow from a vertical surface. Perdikis and Repatis [26] discussed the heat transfer of a micropolar fluid in the presence of radiation. Elbashbeshby and Bazid [27] reported on the radiation effects on the mixed convection flow of a micropolar fluid. Moreover, when the radiative heat transfer takes place, the fluid involved can be electrically conducted in the sense that it is ionized owing to a high operating temperature. Heat and mass transfer in a magneto hydrodynamic micropolar fluid flow through a porous medium under different physical conditions was examined by Ibrahim et al. [28]. Sharma et al. [29-30] discussed the radiation effect on a free convective flow along a uniform moving porous vertical plate in the presence of a transverse magnetic field.

All of the previously mentioned studies assumed no slip boundary conditions. The non-adherence of the fluid to a solid boundary is known as velocity slip. It is a phenomenon that has been observed under certain circumstances [31]. In many engineering applications, the particle adjacent to a solid surface no longer takes the velocity of the surface. The particle at the surface has a finite tangential velocity; it "slips" along the surface. This flow regime is called the slip-flow regime and this effect cannot be neglected. Sharma and Sharma [32] studied the free convection flow past a vertical porous plate with variable suction in the slip-flow regime. Considerably less work has been done concerning the effect of variable fluid properties on a micropolar fluid flow incorporating the effect of velocity slip. Hence, based on the above discussion, the objective of the present work is to study the effect of radiation on mixed convection flow of a magneto- 
micropolar fluid past a vertical porous plate through a porous medium with variable permeability in the slipflow regime.

\section{Formulation of the problem}

Consider an unsteady, two dimensional, mixed convection flow of a micropolar fluid past a semiinfinite vertical plate embedded in a porous medium and subjected to a transverse magnetic field with variable permeability and slip flow regime. The $x^{*}$-axis is taken along the porous plate in the upward direction and $y^{*}$-axis normal to it. Due to the semi-infinite plane surface assumption, the flow variables are functions of $y^{*}$ and $t^{*}$ only. Under these conditions, the governing equations can be written in a Cartesian frame of reference as:

\section{Continuity equation}

$$
\frac{\partial v^{*}}{\partial y^{*}}=0
$$

Linear momentum equation

$$
\begin{aligned}
& \frac{\partial u^{*}}{\partial t^{*}}+v^{*} \frac{\partial u^{*}}{\partial y^{*}}=\left(v+v_{r}\right) \frac{\partial^{2} u^{*}}{\partial y^{* 2}}-\frac{v u^{*}}{K^{*}}-\frac{\sigma}{\rho} B_{0}^{2} u^{*}+2 v_{r} \frac{\partial \omega^{*}}{\partial y^{*}}+ \\
& +g \beta\left(T^{*}-T_{\infty}^{*}\right)+g \beta_{c}\left(C^{*}-C_{0}^{*}\right),
\end{aligned}
$$

Permeability of the medium is assumed to be of the form

$$
K^{*}\left(t^{*}\right)=K^{*}\left(1+\in A e^{n^{*} t^{*}}\right) .
$$

Angular momentum

$$
\rho J^{*}\left(\frac{\partial \omega^{*}}{\partial t^{*}}+v^{*} \frac{\partial \omega^{*}}{\partial y^{*}}\right)=\gamma \frac{\partial^{2} \omega^{*}}{\partial y^{* 2}} .
$$

Energy equation

$$
\frac{\partial T^{*}}{\partial t^{*}}+\mathrm{v}^{*} \frac{\partial T^{*}}{\partial y^{*}}=\frac{\kappa}{\rho C p} \frac{\partial^{2} T^{*}}{\partial y^{* 2}}-\frac{1}{\rho C p} \frac{\partial q_{r}}{\partial y^{*}} .
$$

Mass transfer equation

$$
\frac{\partial C^{*}}{\partial t^{*}}+v^{*} \frac{\partial C^{*}}{\partial y^{*}}=D^{*} \frac{\partial^{2} C^{*}}{\partial y^{* 2}}-K C^{*}
$$

The radiative heat flux term by using Rosseland approximation [33-35] is given by

$$
q_{r}=-\frac{4 \sigma^{*} \partial T^{4}}{3 a_{R} \partial y^{*}}
$$


The boundary conditions suggested by the physics of the problem are

$$
\begin{aligned}
& u^{*}=U_{0}+L_{l}^{*}\left(\frac{\partial u^{*}}{\partial y^{*}}\right), \quad \omega^{*}=-m \frac{\partial u^{*}}{\partial y^{*}}, \quad T^{*}=T_{w}^{*}+\varepsilon\left(T^{*}{ }_{w}-T_{\infty}^{*}\right) e^{n^{*} t^{*}}, \\
& C^{*}=C_{w}^{*}+\varepsilon\left(C^{*}{ }_{w}-C^{*}\right) e^{n^{*} t^{*}} \quad \text { at } \quad y^{*}=0, \\
& u^{*} \rightarrow 0, \quad \omega^{*} \rightarrow 0, \quad T^{*} \rightarrow T_{\infty}^{*}, \quad C^{*} \rightarrow C_{\infty}^{*} \quad \text { as } \quad y^{*} \rightarrow \infty .
\end{aligned}
$$

The boundary conditions for microrotation variables $\omega^{*}$ describe relationships with the surface stress. In this equation, the parameter $m$ is a number between 0 and 1 that relates the microgyration vector to the shear stress. The value $m=0$ corresponds to the case where the particle density is sufficiently large so that microelements close to the wall are unable to rotate. The value $m=0.5$ is indicative of weak concentration and when $m=1$ flows are believed to represent turbulent boundary layers (Rees and Bassom [36]).

From the continuity Eq.(2.1), the suction normal to the plate can be written as

$$
v^{*}=-V_{0}\left(1+\in B e^{n^{*} t^{*}}\right)
$$

where $B$ is a real positive constant with a magnitude less than unity and $V_{0}$ is the scale of suction velocity which has a non-zero positive constant.

It is assumed that the temperature differences within the flow are such that $T^{4^{*}}$ may be expressed as a linear function of the temperature $T$. This is accomplished by expanding $T^{4^{*}}$ in a Taylor series about $T_{\infty}^{*}$ (which is assumed to be independent of the $y$ coordinate) and neglecting higher order terms, it can be expressed as

$$
T^{4 *} \approx 4 T_{\infty}^{3 *}-3 T_{\infty}^{4} *
$$

Using Eqs (2.4) and (2.10) gives

$$
\frac{\partial T^{*}}{\partial t^{*}}+v^{*} \frac{\partial T^{*}}{\partial y^{*}}=\frac{\kappa}{\rho C p} \frac{\partial^{2} T^{*}}{\partial y^{* 2}}+\frac{16 \sigma^{*} T_{\infty}^{*}}{3 \rho C p a_{R}} \frac{\partial^{2} T^{*}}{\partial y^{2}} .
$$

Introduce the following non-dimensional quantities

$$
\begin{aligned}
& u=\frac{u^{*}}{U_{0}}, \quad v=\frac{v^{*}}{V_{0}}, \quad y=\frac{V_{0} y^{*}}{v}, \quad \omega=\frac{v}{U_{0} V_{0}} \omega^{*}, \quad t=\frac{t^{*} V_{0}^{2}}{v}, \\
& \theta=\frac{T^{*}-T_{\infty}^{*}}{T_{w}^{*}-T_{\infty}^{*}}, \quad C=\frac{C^{*}-C_{\infty}^{*}}{C_{w}^{*}-C_{\infty}^{*}}, \quad n=\frac{n^{*} v}{V_{0}^{2}}, \quad \alpha=\frac{K^{*} V_{0}^{2}}{v^{2}}, \quad J=\frac{V_{0}^{2} J^{*}}{v^{2}}, \\
& M=\frac{\sigma B_{0}^{2} v}{\rho V_{0}^{2}} \text { is the magnetic field parameter, }
\end{aligned}
$$


$\mathrm{Gr}=\frac{v \beta_{f} g\left(T_{w}^{*}-T_{\infty}^{*}\right)}{U_{0}^{2} V_{0}^{2}}$ is the Grashof number,

$\mathrm{Gc}=\frac{v g \beta_{c}\left(C_{w}^{*}-C_{\infty}^{*}\right)}{V_{0}^{2} U_{0}}$ is the modified Grashof number,

$\operatorname{Pr}=\frac{v \rho C_{p}}{\kappa}=\frac{v}{\alpha}$ is the Prandtl number,

$\mathrm{Sc}=\frac{\mathrm{v}}{D^{*}}$ is the Schmidt number,

$K_{l}=\frac{K v}{V_{0}^{2}}$ is the chemical reaction parameter,

$R=\frac{\kappa a_{R}}{4 \sigma * T_{\infty}^{*}}$ is the radiation parameter,

$h=\frac{L_{1}^{*} V_{0}}{v}$ is Rarefaction parameter.

Furthermore, the spin-gradient viscosity $\gamma$, which gives some relationship between the coefficient of viscosity and microinertia, is defined as

$$
\gamma=\left(\mu+\frac{1}{2} \Lambda\right) J^{*}=\mu \mathrm{J}^{*}\left(1+\frac{1}{2} B v\right), \quad B v=\frac{\Lambda}{\mu}
$$

where $B v$ denotes the dimensional viscosity ratio, and $\Lambda$ is the coefficient of gyro-viscosity (or vortex viscosity).

In view of Eqs (2.9) - (2.13), the governing Eqs (2.2) - (2.5) reduce to the following dimensionless form

$$
\begin{aligned}
& \frac{\partial u}{\partial t}-\left(1+\in \mathrm{B} e^{n t}\right) \frac{\partial u}{\partial y}=(1+B v) \frac{\partial^{2} u}{\partial y^{2}}+\left[M+\frac{1}{\alpha\left(1+A e^{n t}\right)}\right] \mathrm{u}+2 B v \frac{\partial \omega}{\partial y}+\operatorname{Gr} \theta+\operatorname{Gc} C \\
& \frac{\partial \omega}{\partial t}-\left(1+\in B e^{n t}\right) \frac{\partial \omega}{\partial y}=\frac{1}{\varphi} \frac{\partial^{2} \omega}{\partial y^{2}} \\
& \frac{\partial \theta}{\partial t}-\left(1+\in B e^{n t}\right) \frac{\partial \theta}{\partial y}=\left(\frac{1}{\operatorname{Pr}}+\frac{4}{3 R \operatorname{Pr}}\right) \frac{\partial^{2} \theta}{\partial y^{2}}
\end{aligned}
$$




$$
\frac{\partial \mathrm{C}}{\partial t}-\left(1+\in B e^{n t}\right) \frac{\partial C}{\partial y}=\frac{1}{\mathrm{Sc}} \frac{\partial^{2} C}{\partial y^{2}}-K_{l} C
$$

where $\quad \varphi=\frac{\mu J^{*}}{\gamma}=\frac{2}{2+B v}$,

with corresponding boundary conditions

$$
\begin{aligned}
& u=1+h \frac{\partial u}{\partial y}, \quad \omega=-m \frac{\partial u}{\partial y}, \quad \theta=1+\varepsilon e^{n t}, \quad \mathrm{C}=1+\varepsilon e^{n t} \quad \text { at } \quad y=0, \\
& u \rightarrow 0, \quad \omega \rightarrow 0, \quad \theta \rightarrow 0, \quad C \rightarrow 0 \quad \text { as } \quad y \rightarrow \infty .
\end{aligned}
$$

\section{Numerical solution}

The unsteady, nonlinear coupled Eqs (2.14)-(2.17) with conditions (2.18)-(2.19) are solved by using an explicit finite-difference scheme. Consider a rectangular region with $y$ varying from 0 to $y \max (=6)$, where $y$ max corresponds to $y=\infty$ which lies well outside the momentum and energy boundary layers. The region to be examined in $(y, t)$ space is covered by a rectilinear grid with sides parallel to the $y$ axis and $\Delta t$, the grid spacing in $y$, and $t$ directions, respectively. The grid points $(y, t)$ are given by $(i \Delta y, k \Delta t)$. The finitedifference equations corresponding to (2.14)-(2.17) are given by

$$
\begin{aligned}
& \frac{U_{i}^{k+1}-U_{i}^{k}}{\Delta t}-\left(1+\varepsilon B e^{n t_{i}^{k}}\right) \frac{U_{i}^{k}-U_{i-1}^{k}}{\Delta y}=\frac{U_{i+1}^{k}-2 U_{i}^{k}+U_{i-1}^{k}}{\Delta y^{2}}+\operatorname{Gr} \theta_{i}^{k}+G m \varphi_{i}^{k}+ \\
& +\left[M+\frac{1}{\alpha\left(1+\varepsilon B e^{n t_{i}^{k}}\right)}\right] U_{i}+2 B v\left(\frac{\omega_{i}^{k}-\omega_{i-1}^{k}}{\Delta y}\right), \\
& \frac{\omega_{i}^{k+1}-\omega_{i}^{k}}{\Delta t}-\left(1+\varepsilon B e^{n t_{i}^{k}}\right) \frac{\omega_{i}^{k}-\omega_{i-1}^{k}}{\Delta y}=\frac{1}{\phi} \frac{\omega_{i+1}^{k}-2 \omega_{i}^{k}+\omega_{i-1}^{k}}{\Delta y^{2}}, \\
& \frac{\theta_{i}^{k+1}-\theta_{i}^{k}}{\Delta t}-\left(1+\varepsilon B e^{n t_{i}^{k}}\right) \frac{\theta_{i}^{k}-\theta_{i-1}^{k}}{\Delta y}=\left[\frac{1}{\operatorname{Pr}}+\frac{4}{3 R \operatorname{Pr}}\right] \frac{\theta_{i+1}^{k}-2 \theta_{i}^{k}+\theta_{i-1}^{k}}{\Delta y^{2}}, \\
& \frac{C_{i}^{k+1}-C_{i}^{k}}{\Delta t}-\left(1+\varepsilon B e^{n t_{i}^{k}}\right) \frac{C_{i}^{k}-C_{i-1}^{k}}{\Delta y}=\frac{1}{\mathrm{Sc}} \frac{C_{i+1}^{k}-2 C_{i}^{k}+C_{i-1}^{k}}{\Delta y^{2}}-K_{1} C_{i}^{k} .
\end{aligned}
$$


The finite-difference equations at every internal nodal point on a particular $n$-level constitute a tridiagonal system of equations. These equations are solved by using the Thomas algorithm [37]. Computations are carried out until the steady-state solution is assumed to have been reached when the absolute difference between the values of velocity as well as temperature at two consecutive time steps are less than $10^{-7}$ at all grid points.

\section{Results and discussion}

In order to get a physical insight into the problem, the numerical calculations for the velocity, microrotation, temperature and concentration profiles, the rate of heat transfer and mass transfer across the boundary layer for various values of the parameter have been carried out. The effects of the main controlling parameters as they appear in the governing equations are discussed in the current section. In this study, entire numerical calculations have been performed with $\varepsilon=0.01, n=1$ and $t=1$ while $\operatorname{Pr}, R, \mathrm{Sc}, \mathrm{Gr}, \mathrm{Gc}, B v, M, \alpha$, $h, m, B, K_{l}$ and $A$ are varied over ranges, which are listed in the figure legends. The velocity profile $u$ is plotted in Fig. 1 for different values of the radiation parameter $(R)$, rare factor parameter $(h)$, viscosity ratio parameter $\left(B_{v}\right)$, permeability parameter $(\alpha)$ and chemical reaction parameter. The figure reveals that on increasing the values of the permeability parameter $(\alpha)$ and chemical reaction parameter the velocity profile increase across the boundary layer while a reverse effect is observed for the rare factor reaction parameter. It is noted that the velocity $u$ decreases as the radiation parameter $(R)$ and rare factor parameter $(h)$ increase. This result can be explained by the fact that a decrease in the radiation parameter $R=k a_{R} / 4 \sigma^{*} T^{3}$ for given $k$ and $T \infty$, means a decrease in the Rosseland radiation absorbtivity $\left(a_{R}\right)$. In view of Eqs (2.4) and (2.6), it is concluded that the divergence of the radiative heat flux $\partial q_{r} / \partial y^{*}$ increases as $a_{R}$ decreases and this means that the rate of radiative heat transferred to the fluid increases and consequently the fluid temperature and hence the velocity of its particles also increases. The velocity distribution decreases with increasing $B_{v}$. The phenomenon reflects the fact that the effect of increase in the value of $B_{v}$ will result in an enhancement of the total viscosity in the fluid flow because $B_{v}$ is directly proportional to vortex viscosity which makes the fluid flow more viscous and so weakens the convection currents. In addition, the magnitude of $\omega$ increases as $B_{v}$ increases.

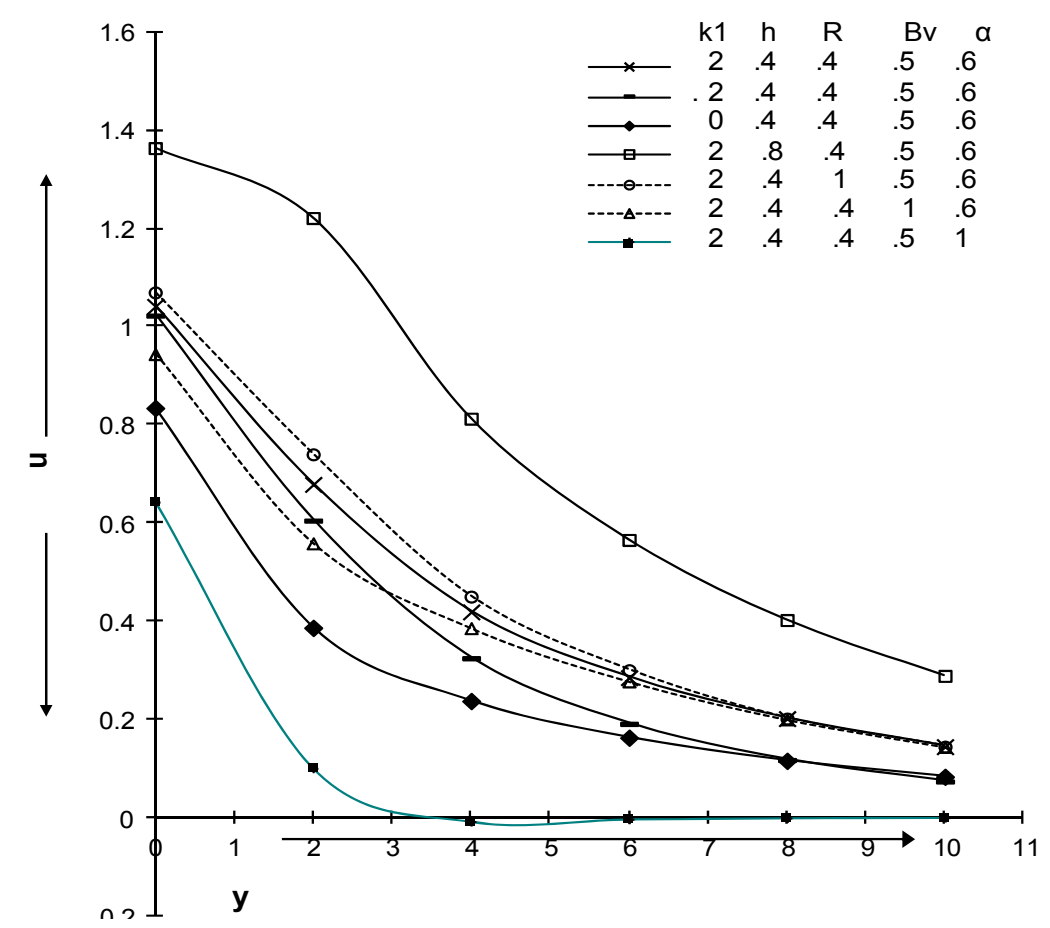

Fig.1. Velocity profiles $u$ for $\operatorname{Pr}=1, M=1, \mathrm{Gr}=2, m=.5, \mathrm{Sc}=.22, B=.5$. 
The microrotation profile against span-wise coordinate $y$ for different values of the radiation parameter $(R)$, rare factor parameter $(h)$, viscosity ratio parameter $\left(B_{v}\right)$, permeability parameter $(\alpha)$ and chemical reaction parameter is shown in Fig.2. It is noticed that the magnitude of the angular velocity $\omega$ decreases as the radiation parameter $(R)$ and rare factor parameter $(h)$ increase. The figure reveals that on increasing the values of the permeability parameter $(\alpha)$ and chemical reaction parameter, the magnitude of $\omega$, across the boundary layer, tends to decrease. It is also observed that the microrotation profile increases with increasing the viscosity ratio parameter $\left(B_{v}\right)$.

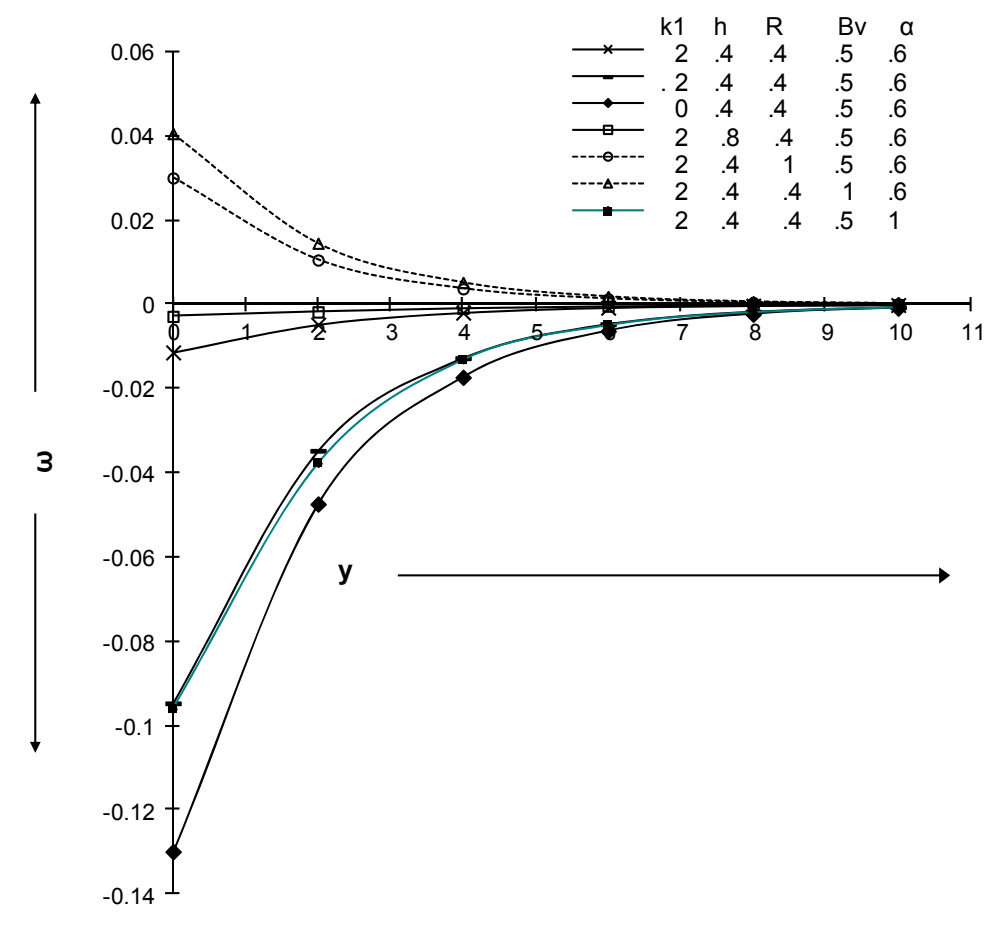

Fig.2. Micro rotation profiles $\omega$ for $\operatorname{Pr}=1, M=1, \mathrm{Gr}=2, m=.5, \mathrm{Sc}=.22, B=.5$.

Since the velocity and micro-rotation profiles in the boundary layer are known, the skin friction coefficient $\left(C_{f}\right)$ at the wall is given by

$$
C_{f}=\frac{2 T_{w}^{*}}{\rho U_{0} V_{0}}=2[1+(1-n) \beta] u^{\prime}(0),
$$

the couple stress coefficient $\left(C_{m}\right)$ at the plate is written as

$$
C_{m}=\frac{M_{w}}{\mu J U_{0}}=\left(1+\frac{\beta}{2}\right) w^{\prime}(0) .
$$

Figures 3 and 4 illustrate the variations of the skin friction coefficient $C_{f}$ and couple stress coefficient $\mathrm{Cm}$ for various values of $h, R, B_{v}, K_{l}$ and $\alpha$ against $\mathrm{Gr}$. It is observed that higher values of the radiation parameter, chemical reaction parameter and rarefaction parameter lead to a reduction in the skin friction coefficient and couple stress coefficient. It is noted that $C_{f}$ and $C_{m}$ decrease with increasing $B_{v}$ for small values of Gr. It is concluded that $C_{f}$ and $C_{m}$ increase with increasing the values of $\alpha$. Further, $C_{f}$ and $C_{m}$ increase as the buoyancy parameter Gr increases. The reason for this is that an increase in the buoyancy effect in a mixed convection flow leads to an acceleration of the fluid flow, which increases the friction factor. 
Figure 5 depicts the temperature profiles $\theta$ along the span-wise coordinate $y$ for different values of the Prandtl number $\left(\operatorname{Pr}=0.71\right.$, for air at $20^{\circ} \mathrm{C}$ and 1 atmospheric pressure, $\operatorname{Pr}=1.0$ for electrolytic solution, at $20^{\circ} \mathrm{C}$ and 1 atmospheric pressure), radiation parameter $(R)$ and suction parameter $(B)$. The figure indicates that the temperature reduces with an increase in the radiation parameter $(R)$ and suction parameter $(B)$ for air while a reverse effect is observed for an electrolytic solution. The numerical results show that the temperature decreases with an increase in the Prandtl number. This is due to the fact that a fluid with a high Prandtl number has a relatively low thermal conductivity which results in the reduction of the thermal boundary layer thickness.

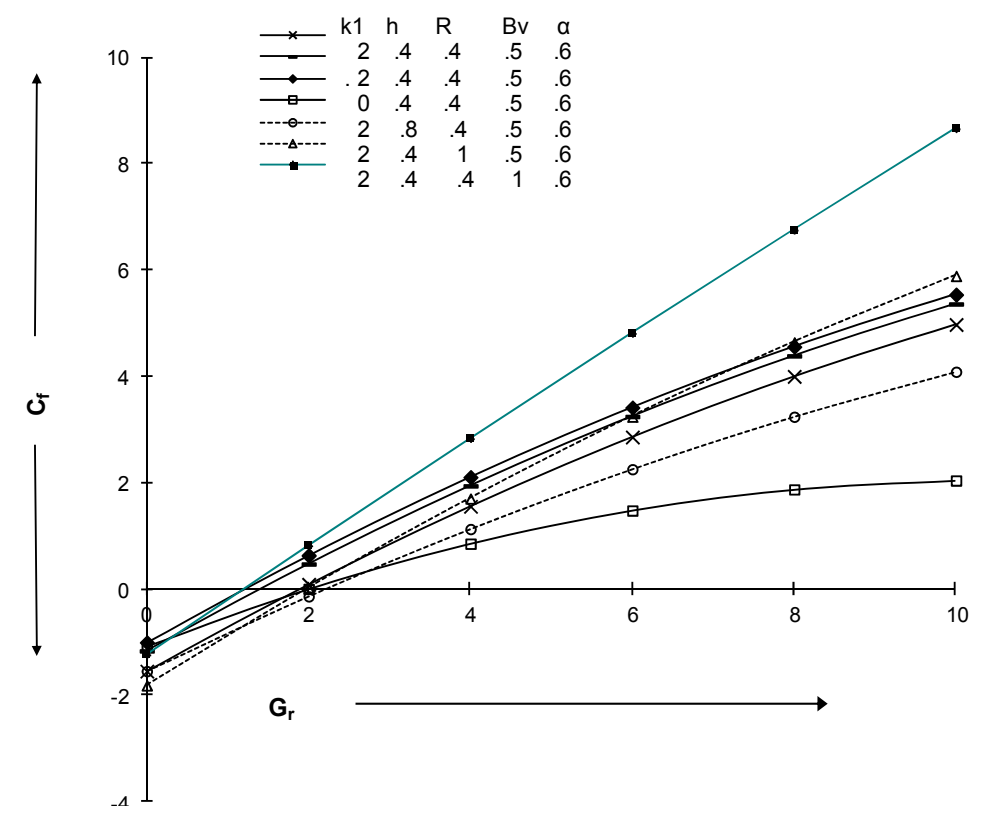

Fig.3. Skin-friction coefficient for $\operatorname{Pr}=1, M=1, m=.5, \mathrm{Sc}=.22, B=.1$.

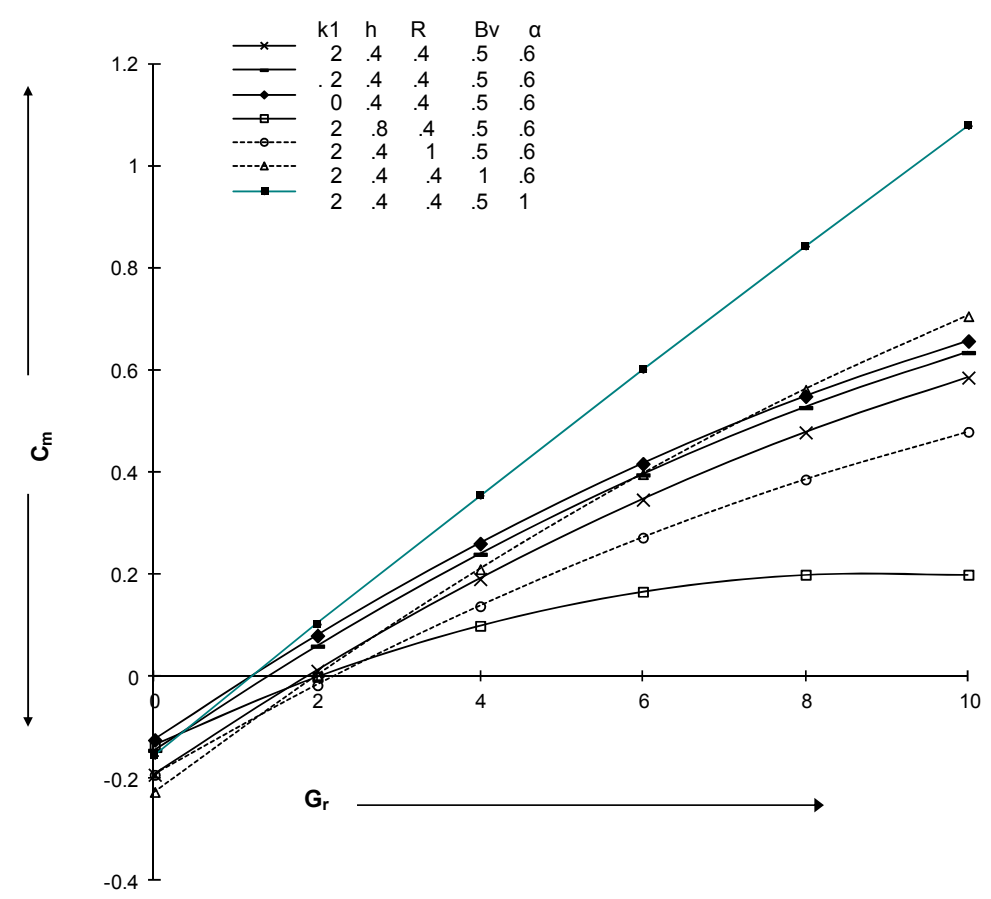

Fig.4. Couple stress coefficient for $\operatorname{Pr}=1, M=1, m=.5, \mathrm{Sc}=.22, B=.1$. 


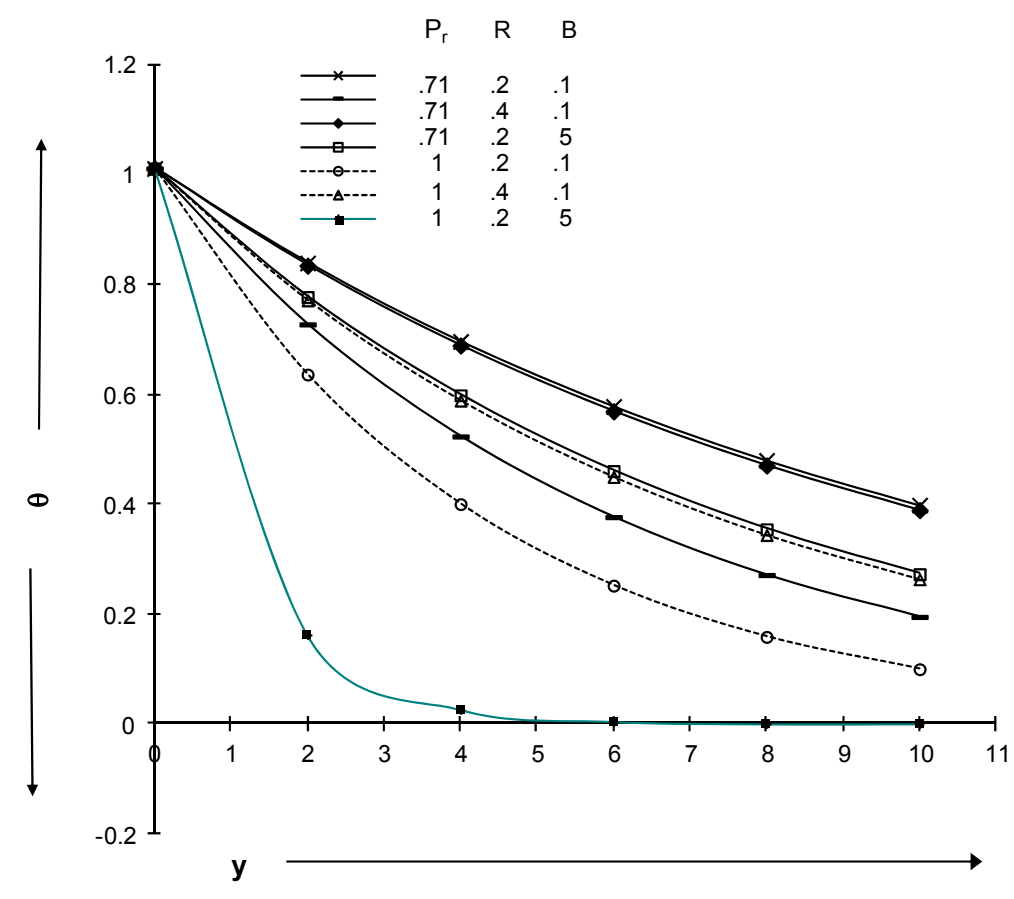

Fig.5. Temperature profiles for $\varepsilon=0,1, n=.1, t=1$.

The rate of heat transfer in terms of the Nusselt number $(\mathrm{Nu})$ is given by

$$
\mathrm{Nu}=-\left.\frac{\partial \theta}{\partial y}\right|_{y=0} .
$$

The rates of heat transfer for different values of the Prandtl number $(\operatorname{Pr})$ and radiation parameter $(R)$ taking $\varepsilon=0.01, n=1$ and $t=1$ are presented in Fig.6. It is observed that the heat transfer rate is enhanced as the Prandtl number and radiation parameter increase. It is also noted that the Nusselt number increases with an increase in the radiation parameter $R$.

Concentration profiles for different gases are shown in Fig.7. The values of Schmidt number (Sc) are chosen to represent the most common diffusing chemical species like hydrogen $(\mathrm{Sc}=0.22)$, oxygen $(\mathrm{Sc}=$ $0.66)$, and ammonia $(\mathrm{Sc}=0.78)$ at a temperature of $25^{\circ} \mathrm{C}$ and 1 atmospheric pressure. A comparison of curves in the figure shows a decrease in the concentration distribution $C(y)$ with an increase in the Schmidt number because the smaller values of Sc are equivalent to increasing the chemical molecular diffusivity $(D)$. Hence, the concentration of the species is higher for small values of Sc and lower for larger values of Sc. The concentration profiles also decrease with an increase in the suction parameter $(B)$. The concentration profiles attain their maximum values at the wall and decrease exponentially with $y$ and finally tend to zero as $y \rightarrow \infty$. The rate of mass transfer in terms of the Sherwood number (Sh) is given by

$$
\mathrm{Sh}=\frac{J^{*} v}{V_{0} \rho D^{*}\left(C_{w}^{*}-C_{\infty}^{*}\right)}=-\left.\frac{\partial C}{\partial y}\right|_{y=0} .
$$

Figure 8 depicts the rate of mass transfer in terms of Sherwood number (Sh) at the porous plate for hydrogen $(\mathrm{Sc}=0.22)$, oxygen $(\mathrm{Sc}=0.66)$ and ammonia $(\mathrm{Sc}=0.78)$ at a temperature $25^{\circ} \mathrm{C}$ and 1 atmospheric pressure taking $\varepsilon=0.01, n=1$ and $t=1$. It is clear that increasing values of the Schmidt number enhance the 
mass transfer rate. Also, the figure indicates that the rate of mass transfer increase with increasing values of the chemical reaction parameter.

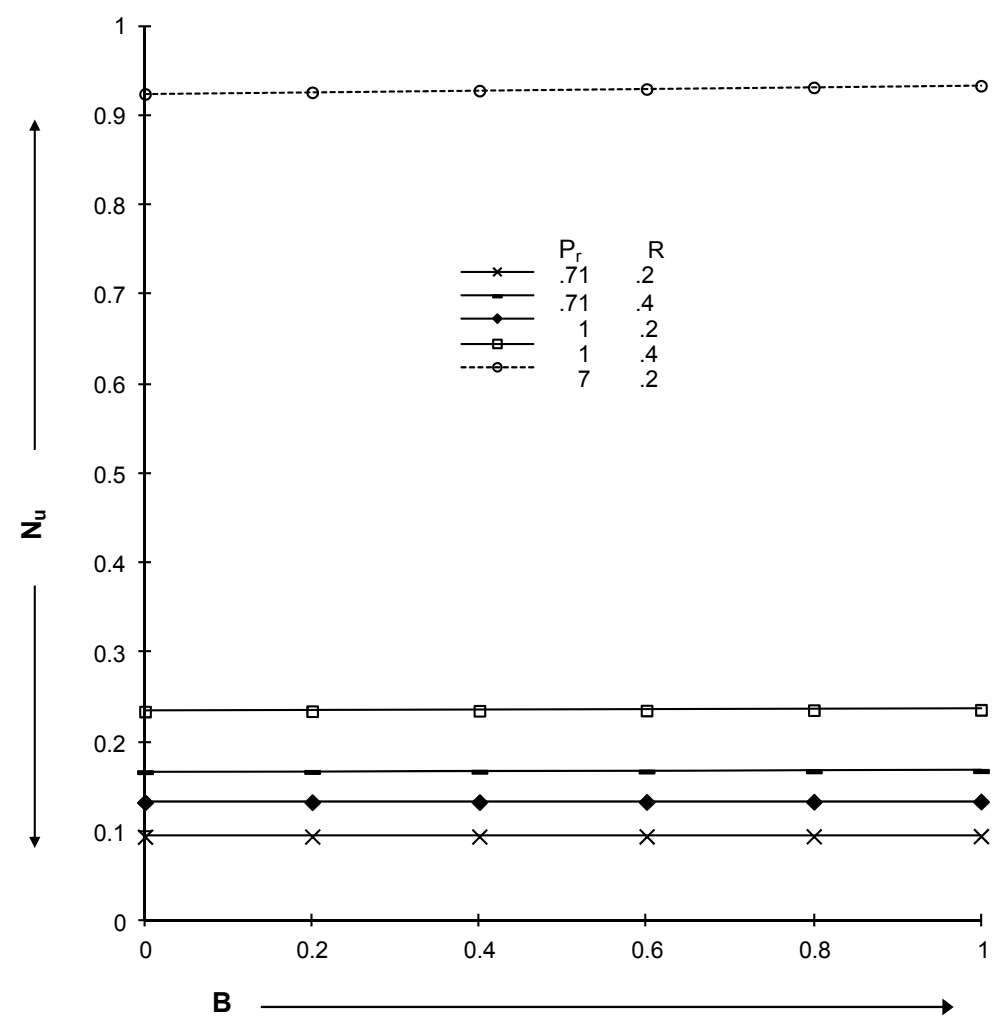

Fig.6. Rate of heat transfer for $\varepsilon=0.01, n=0.1, t=1$.

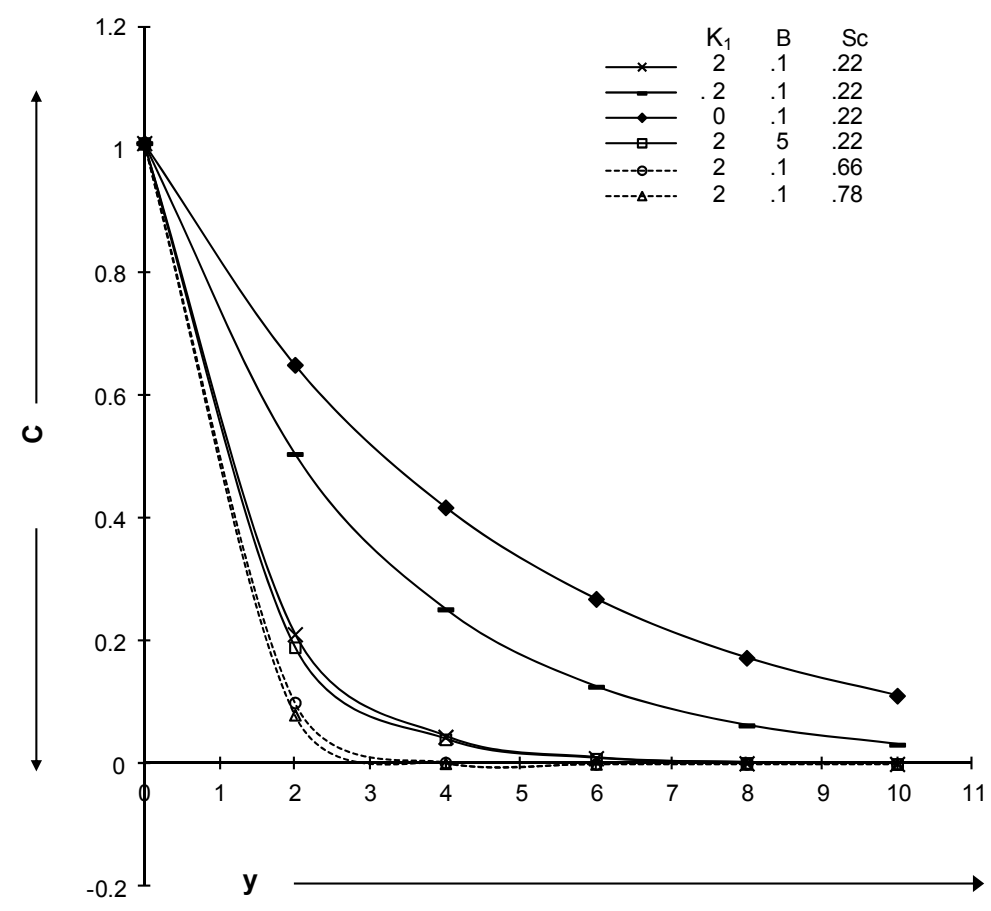

Fig.7. Concentration profiles for $\varepsilon=.01, n=.1, t=1$. 


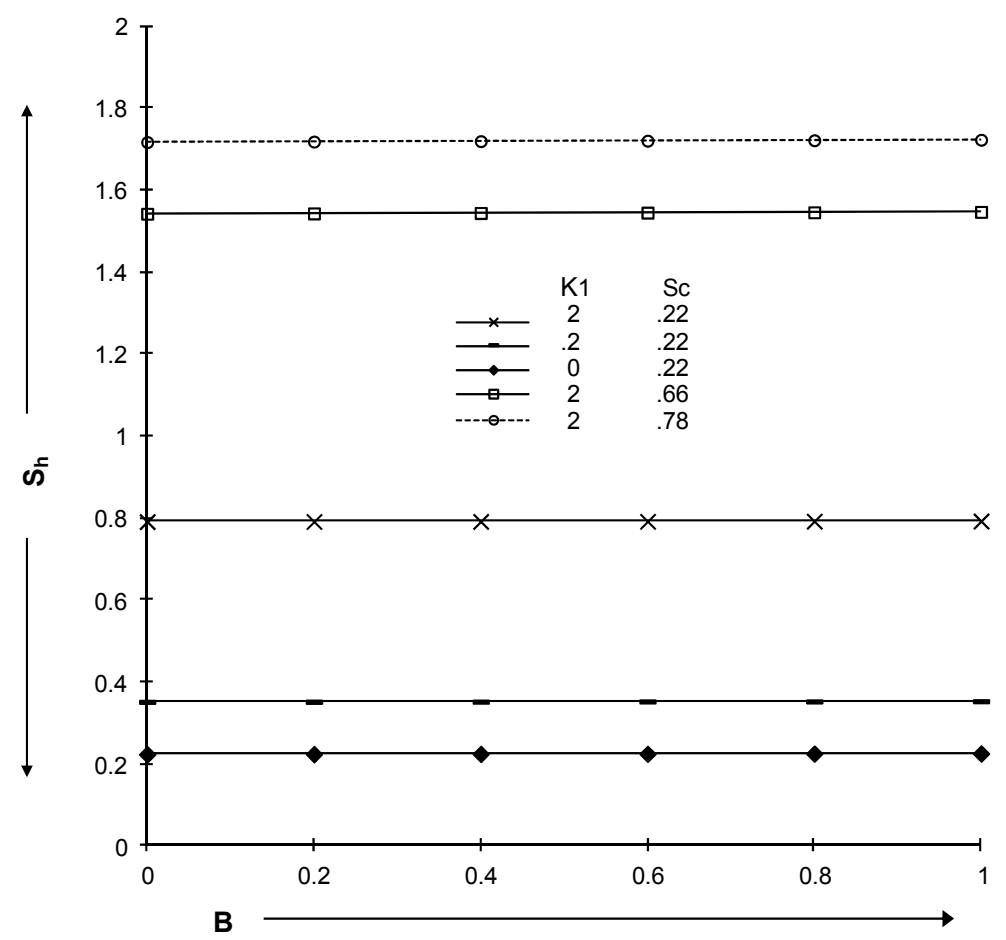

Fig.8. Rate of mass transfer for $\varepsilon=0.01, n=0, t=1$.

\section{Nomenclature}

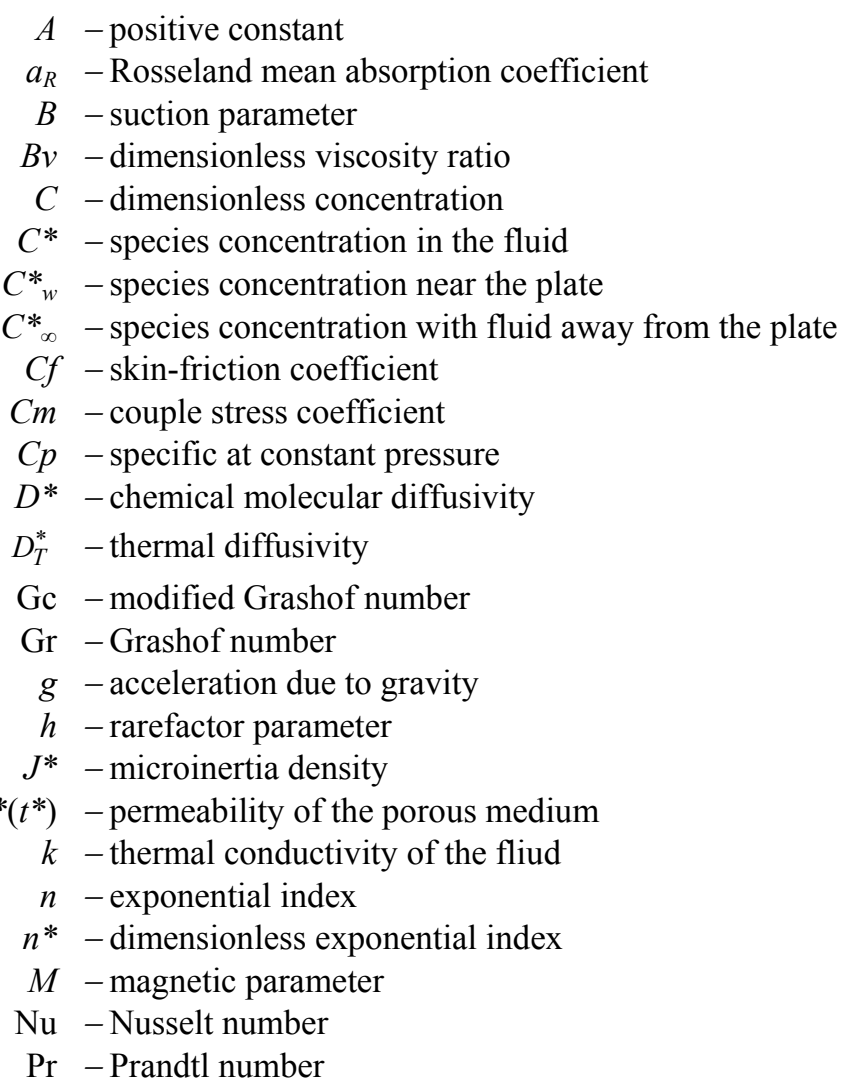


$R$ - radiation parameter

Sc - Schmidt number

Sh - Sherwood number

$T^{*}$ - temperature of the fluid near the plate

$T^{*} w$ - temperature of the plate

$T^{*} \infty$ - temperature of the fluid far away from the plate

$t$ - time

$t^{*}-$ dimensionless time

UO - plate velocity in the direction of flow

$U^{*}, v^{*}$ - components of dimensional velocities along $x^{*}$ and $y^{*}$ directions, respectively

$u, v$-dimensionless velocities along $x$ and $y$ direction respectively

$x, y$-dimensionless Coordinate axis along and normal to the porous plate, respectively

$x^{*}, y^{*}$-coordinate axis along and normal to the porous plate, respectively

$\alpha$-dimensionless permeability parameter

$\beta-$ coefficient of volumetric thermal expansion of the fluid

$\beta c$ - volumetric coefficient of expansion with concentration

$\gamma$ - spin- gradient viscosity

$\theta$-dimensionless temperature

$\Lambda$ - vortex viscosity

$\mu-$ dynamic viscosity

$v$ - kinematic viscosity

$v r$ - kinematic rotational viscosity

$\rho$ - density

$\sigma$ - electrical conductivity of the fluid

$\sigma^{*}$ - Stefan-Boltzmann constant

$\Omega^{*} \quad$ - component of the angular velocity vector normal to the $x *-y *$ plane

$\omega$-dimensionless component of the angular velocity vector normal to the $x-y$ plane

\section{Subscripts}

$w$ - wall condition

$\infty-$ free stream condition.

\section{Superscripts}

* - dimensional properties

\section{References}

[1] Eringen A.C. (1964): Simple microfluids. - Int. J. Engng. Sci., vol.2, pp.205-217.

[2] Eringen A.C. (1972): Theory of Termomicrofluids. - J. Math. Anal. Appl., vol.38, pp.480-496.

[3] Gorla R.S.R. (1992): Mixed convection in a micropolar fluid from a vertical surface with uniform heat flux. - Int. J. Engng. Sci., vol.30, pp.349-358.

[4] Rees D.A.S. and Pop I. (1998): Free convection boundary layer flow of a micropolar fluid from a vertical flat plate. - IMAJ. Appl. Math., vol.61, pp.179-197.

[5] Takhar H.S. and Soundalgekar V.M. (1980): Heat transfer in a flat plate flow of a micropolar fluid. - Rheologica Acta, vol.19, pp.525-526.

[6] Takhar H.S. and Soundalgekar V.M. (1985): Flow and heat transfer of micropolar fluid past a porous plate. Indian J. Pure Appl. Math., vol.16, pp.552-558. 
[7] Gorla R.S.R., Slaouti A.S. and Takhar H.S. (1998): Free convection in micropolar fluids over a uniformly heated vertical plate. - Int. J. Heat and Fluid Flow, vol.8, pp.504-518.

[8] Gorla R.S.R., Mohammedan A., Mansour M. and Hussein I. (1995): Unsteady natural convection from a heated vertical plate in micropolar fluid. - Numerical Heat Transfer, Part A, vol.28, pp.253-262.

[9] Sharma K., Chaudhary R.C. and Sharma P.K. (2007): Fluctuating mass transfer on three-dimensional flow through a porous medium with variable permeability. - Advances in Theoretical and Applied Mathematics, vol.2, No.3, pp.257-267.

[10] Raptis A. and Takhar H.S. (1999): Polar fluid through a porous medium. - Acta Mechanica, vol.135, pp.91-93.

[11] Helmy K.A. (1998): MHD unsteady free convection flow past a vertical porous plate. - ZAMM, vol.98, pp.255270.

[12] Sharma K. and Chaudhary R.C. (2008): Hydromagnetic unsteady mixed convection and mass transfer flow past a vertical porous plate immersed in a porous medium with Hall effect. - Engineering Transactions, vol.56, No.1, pp.3-23.

[13] Chaudhary R.C. and Sharma B.K. (2006): Combined heat and mass transfer by laminar mixed convection flow from a vertical surface with induced magnetic field. - Journal of Applied Physics, vol.99, pp.34901-10.

[14] El-Hakiem M.A., Mohammadein A.A., El-Kabeir S.M.M. and Gorla R.S.R. (1999): Joule heating effects on magnetohydrodynamic free convection flow of a micropolar fluid. - Int. Comm. Heat Mass Tran., vol.26, No.2, pp.219-227.

[15] El-Amin M.F. (2001): Magnetohydrodynamic free convection and mass transfer flow in micropolar fluid with constant suction. - J. Magn. Mater., vol.234, pp.567-574.

[16] Kim Y.J. (2001): Unsteady convection flow of micropolar fluids past a vertical plate embedded in a porous medium. - Acta. Mech., vol.148, pp.105-116.

[17] Kim Y.J. (2004): Heat and mass transfer in MHD micropolar flow over a vertical moving plate in a porous medium. - Trans. Porous Media, vol.56, pp.17-37.

[18] Kim Y.J. and Lee J.C. (2003): Analytical studies on MHD oscillatory flow of a micropolar fluid over a vertical porous plate. - Surface and Coatings Technology, vol.171, pp.187-193.

[19] Sharma K., Mishra A. and Gupta S. (2013): Heat and mass transfer in magneto-biofluid flow through a nonDarcian porous medium with Joule effect. - J. Eng. Phys. and Thermo Phys., vol.86, No.4, pp.716-725.

[20] Das U.N., Deka R.K. and Soundalgekar V.M. (1994): Effects of mass transfer on flow past an impulsively started infinite vertical plate with constant heat flux and chemical reaction. - Forschung im Engenieurwesen Engineering Research Co., pp.284-287.

[21] Sharma B.K., Singh A.P., Yadav K. and Chaudhary R.C. (2013): Effects of chemical reaction on magnetomicropolar fluid flow from a radiative surface with variable permeability. - International Journal of Applied Mechanics and Engineering, vol.18, No.3, pp.833-85.

[22] Sharma B.K., Yadav K., Mishra N.K. and Chaudhary R.C. (2012): Soret and Dufour effects on unsteady MHD mixed convection flow past a radiative vertical porous plate embedded in a porous medium with chemical reaction. - Applied Mathematics, vol.3, No.7, pp.717-723. doi:10.4236/am.2012.37105.

[23] Muthucumarswamy R. and Ganesan P. (2001): First order chemical reaction on flow past an impulsively started vertical plate with uniform heat and mass flux. - Acta. Mech., vol.147, pp.45-57.

[24] Sharma B.K., Jha A.K. and Chaudhary R.C. (2006): Radiation effect with simultaneous thermal and mass diffusion in MHD mixed convection flow from a vertical surface with Ohmic heating. - Romania Journal of Physics, vol.51, No.7-8, pp.715-727. 
[25] Sharma B.K., Agarwal M. and Chaudhary R.C. (2006): MHD fluctuating free convective flow with radiation embedded in porous medium having variable permeability and heat source/sink. - Journal of Technical Physics, vol.47, No.1, pp.47-58.

[26] Perdikis C. and Raptis A. (1996): Heat transfer of a micropolar fluid by the presence of radiation. - Heat and Mass Transfer, vol.31, No.6, pp.381-382.

[27] Elbashbeshy E.M.A. and Bazid M.A.A. (2000): Effect of radiation on forced convection flow of a micropolar fluid over a horizontal plate. - Can. J. Phys./Rev. Can. Phys., vol.78, No.10, pp.907-913.

[28] Ibrahim F.S., Hassanien I.A. and Bakr A.A. (2004): Unsteady magneto-hydrodynamic micropolar fluid flow and heat transfer over a vertical porous plate through a porous medium in the presence of thermal and mass diffusion with a constant heat source. - Can. J. Phys./Rev. Can. Phys., vol.82, No.10, pp.775-790.

[29] Sharma B.K., Chaudhary R.C. and Agarwal M. (2007): Radiation effect on temperature distribution in threedimensional Couette flow with injection or suction. - Applied Mathematics and Mechanics, vol.28, No.3, pp.309-316.

[30] Sharma B.K., Chaudhary R.C. and Agarwal M. (2008): Radiation effect on steady free convective flow along a uniform moving porous vertical plate in presence of heat source/sink and transverse magnetic field. - Bull. Cal. Math. Soc., vol.100, pp.529-538.

[31] Yoshimura A. and Prudhomme R.K. (1998): Wall slip corrections for Couette and parallel disc viscometers. - J. Rheol., vol.32, pp.53-67.

[32] Sharma P.K. and Sharma B.K. (2004): Influence of variable suction on unsteady free convective flow from a vertical flat plate and heat transfer in slip-flow regime. - Ganita Sandesh, vol.18, No.1, pp.55-62.

[33] Raptis A. and Massalas C.V. (1998): Magnetohydrodynamic flow past a plate by the presence of radiation. - Heat and Mass Transfer, vol.34, pp.107-109.

[34] Perdikis C. and Raptis E. (2006): Unsteady MHD flow in the presence of radiation. - Int. J. of Applied Mechanics and Engineering, vol.11, No.2, pp.383-390.

[35] Raptis A. and Perdikis C. (1998): Viscoelastic flow by the presence of radiation. - Z. Angew. Math. Mech., vol.78, No.4, pp.277-279.

[36] Rees D.A.S. and Bassom A.P. (1996): The Blasisum boundary layer flow of microppolar fluid. - Int. Engng. Sci., vol.34, pp.113-124.

[37] Hoffman J.D. (1992): Numerical Methods for Engineers and Scientists. - New York: McGraw-Hill.

Received: July 3, 2016

Revised: April 28, 2017 